\title{
The Synchronous Prevalence of Colorectal Neoplasms in Patients with Stomach Cancer
}

\author{
Ryung Ah Lee \\ Division of Coloproctology, Department of Surgery, Ewha Womans University School of Medicine, Seoul, Korea
}

See Article on Page 246-251

Stomach cancer is still the most common malignancy in Korea in spite of its dramatically decreasing tendency during the last two decades. More importantly, the incidence of colorectal cancer has been increasing during the same period, so clinicians frequently experience synchronous or metachronous stomach cancer with colorectal cancer.

Several reports on colorectal cancer with stomach cancer have been published in Asian countries. In 2010, Yoon et al. [1] reported clinicopathologic data for colorectal cancer with synchronous and metachronous stomach cancer. They reviewed 8,680 patients who underwent surgery for primary sporadic colorectal cancer from 1989 to 2008. They revealed that the incidences of synchronous and metachronous gastric cancer were 0.93 and $1.4 \%$, respectively (combined $2.4 \%$ ). The standardized incidence ratio was 1.199 (95\% confidence interval, 1.005 to 1.420 ). This result is slightly lower than the data for Japan [2] published in 1999. However, Eom et al. [3] reported that the incidence of synchronous or metachronous cancer with gastric cancer was 3.4\% based on data for 4,593 patients who underwent gastric cancer surgery. The most common other primary cancer was colorectal cancer $(20.1 \%)$, followed by lung cancer and liver cancer. Actually, this result is little different from the results published in other papers. Wu et al. [4] analyzed 99 multiple primary cancers with gastric cancer in Taiwan. In the 77 male patients, prostate cancer was the most common (19.5\%), followed by cancers of the colon (18.2\%) and liver (14.3\%). In the 22 female patients, colon cancer was the most common (31.9\%), followed by breast and cervix cancers (22.7\%). Such data revealed the importance of colorectal

Correspondence to: Ryung Ah Lee, M.D.

Department of Surgery, Ewha Womans University Mokdong Hospital,

911-1 Mok-dong, Yangcheon-gu, Seoul 158-710, Korea

Tel: +82-2-2650-5273, Fax: +82-2-2644-7984

Email: ralee@ewha.ac.kr

(c) 2011 The Korean Society of Coloproctology

This is an open-access article distributed under the terms of the Creative Commons Attribution NonCommercial License (http://creativecommons.org/licenses/by-nc/3.0) which permits unrestricted noncommercial use, distribution, and reproduction in any medium, provided the original work is properly cited. cancer screening because colorectal cancer is second common cancer occurring with stomach cancer.

In Korea, Lim et al. [5] reported a prospective study that analyzed the esophagogastroduodenoscopy results from 1,542 consecutive colorectal cancer patients. In their series, synchronous gastric cancers were detected in 31 cases (2.0\%). Of these 31 cases, 26 had early gastric cancer (EGC, 83.9\%) and 5 had advanced gastric cancer. Ten (38.5\%) of the 26 EGC cases were managed using an endoscopic mucosal resection. Compared to colorectal cancer patients without synchronous gastric cancer, the group of patients with synchronous gastric cancer was older $(65.5+/-9.6$ vs. 58.4 $+/-11.3$ years, $\mathrm{P}=0.001$ ) and had a greater proportion of males ( 77.4 vs. $59.4 \%, \mathrm{P}=0.043$ ).

In this research from July 2005 to June 2010, the authors included a total of 123 patients with stomach cancer (86 male), and 246 consecutive, age- and sex-matched persons without stomach cancer were analyzed. All of them underwent a colonoscopy within 6 months after undergoing a gastroscopy. The prevalence of colorectal neoplasms was significantly higher in the stomach cancer group $(35.8 \%)$ than in the control group $(17.9 \%, \mathrm{P}<0.001)$. This paper reports on a kind of case-control study, but still had a weak point of no randomization and retrospective analysis. Also, the authors analyzed all kinds of neoplasms in the colon, including tubular adenomas, villous adenomas and adenocarcinomas. The incidences of pure adenocarcinomas were $6.8 \%$ and $0 \%$ in the stomach cancer group and the normal control group, respectively. Another weak point is the small size of materials compared to other reports. We have to be cautious in accepting the statistical results reported in this paper.

The biology of stomach cancer is apparently different from that of colorectal cancer, as are the risk factors for occurrence and the incidence of relapse. A larger-scale epideiologic study would be essential before concluding that the incidence of colorectal cancer in patients with stomach cancer is higher than it is in the general population.

\section{REFERENCES}

1. Yoon SN, Oh ST, Lim SB, Kim TW, Kim JH, Yu CS, et al. Clinicopathologic characteristics of colorectal cancer patients with syn- 
chronous and metachronous gastric cancer. World J Surg 2010;34: 2168-76.

2. Kaneko S, Yamaguchi N. Epidemiological analysis of site relationships of synchronous and metachronous multiple primary cancers in the National Cancer Center, Japan, 1962-1996. Jpn J Clin Oncol 1999;29:96-105.

3. Eom BW, Lee HJ, Yoo MW, Cho JJ, Kim WH, Yang HK, et al. Synchronous and metachronous cancers in patients with gastric can- cer. J Surg Oncol 2008;98:106-10.

4. Wu CW, Lo SS, Chen JH, Hsieh MC, Li AF, Lui WY. Multiple primary cancers in patients with gastric cancer. Hepatogastroenterology 2006;53:463-7.

5. Lim SB, Jeong SY, Choi HS, Sohn DK, Hong CW, Jung KH, et al. Synchronous gastric cancer in primary sporadic colorectal cancer patients in Korea. Int J Colorectal Dis 2008;23:61-5. 